\title{
Preface to the 1991 Edition
}

WHEN I SET OUT to review the text of this book, twenty years after the original publication, I wondered how many changes would be needed to bring it up to the ways in which the practice of analytical psychology has evolved. Many contributions in the fields of preoedipal dynamics and feminine psychology have enriched our clinical scope.

Under the impact of the sexual revolution and the drug culture, formerly rigid persona and superego-determined ego structures have softened and, at times, even vanished. In Freud's and Jung's days the repression of sexuality and spirituality by an overrigid ego accounted for the majority of psychopathology. Nowadays the problems lie more frequently with insufficient ego structuring, confusion and borderline pathology and the difficulties of finding new forms of I-Thou relationships in a world no longer regulated by viable collective standards.

During Jung's lifetime the main analytic thrust was toward opening a too rigidly structured ego to a dialogue with the nonpersonal psyche. His stress was on the necessity of the ego's confronting and coming to terms with the world of archetypes. Today, perhaps owing to the dissolution of traditional standards, and the consequent weakening of persona support as well as the influx of often inadequately understood Eastern religious and cult influences, we encounter many more instances of inadequate egos being "lost" in the world of archetypes. An adequate personal ego structure has to be built before the transpersonal unconscious can be faced. To this end, introversion alone does not suffice. Working on one's relationships to partners, group and community is an equally important aspect of ego building and individuation.

In response to this situation various "schools" of Jungian practice have developed. The "developmental" school (Andrew Samuels, Jung and the Post Jungians, London: Routledge \& Kegan Paul, 1985, p. 15) tends to promulgate the "clinical" approach that emphasizes the reductive dealing with personal traumatic, early childhood causation over the a priori archetypal data of the structuring and guiding Self. The "classical" and "archetypal" schools, in turn, tend to concern themselves more with archetypal than developmental dynamics and with the analysis of transference and countertransference. In their extreme forms both positions tend to 
identify with one aspect of what is in fact an interdependent field of complementary dynamics.

From this interdependent field perspective I see transformation and healing brought about through actualizing the guiding impulses from the archetypal world and the Self. They operate in terms of one's personal difficulties and emotional and relationship problems and in the effects of past traumatizations. From this perspective individual life can be seen as a dramatic story or "myth" which "stages" dramatic performances in phases that involve "quantum leaps" of creation and breakdown: birth, death and rebirth. Such a model can include both the "clinical" causal view of problems and pathology as "caused" by traumatic "accidents" that interrupted a purportedly "normal" state; and we can also see these traumatic cause-and-effect relations as subordinated to and "necessitated" by the "intents" of the play staged by the individual Self. To be an actor in one's life's drama without understanding its dramatic thrust, the action's place and function within the overall intended order which is both "because" and "in order to," makes it meaningless at best and chaotic at worst. Yet in order to grasp the crisis and its possible resolution, we must understand the meaning of the action as it unfolds as well as the impasse out of which it arose. The dramatic action evolving from the original difficulties must be reconstructed by reductive analysis to make the repressed traumatic conditionings adequately conscious. They must be actualized in the experiences of transference and countertransference. Their meaning or significance, however, arises through archetypal and mythological understanding.

What has meaning can be better borne. When we can forge our experiences of pain into a meaningful pattern, they can be seen as functional elements of our destiny. Chaotic suffering is unbearable. From this perspective we can understand the apparent paradox that digging up and reactivating old and even forgotten misery can have healing effects. And we can also appreciate the experiences of negative as well as positive transference.

A lack of relation to the archetypal dimension results in spiritual impoverishment and a sense of meaninglessness in life. But insufficient anchoring of the archetypal in the personal realm results in mere "head trips" and narcissistic preoccupation. There we merely speculate about archetypal meaning rather than try to discover this meaning through living concretely the prosaic and "trivial" problems and difficulties of everyday feelings and relationships-including those that arise in the transferential interaction with one's therapist. Then the symbol fails to heal and may, indeed, insulate us from the unconscious rather than connect us to it.

While the original edition of this book did not yet explicitly deal with the questions arising from these shifts of accent, its basic viewpoints by 
and large happen to do justice to them. However, I feel that in addition to minor textual changes, there are three areas in which, under the pressure of clinical experience, our concepts have evolved since their original formulation.

One concerns the definitions of the gender archetypes in exclusively contrasexual terms. These have been found to be too limiting by some therapists, this author included. Evidence seems to be accumulating that anima and animus may be seen as archetypal gender potentials, both of which apply to both sexes.

The prime emphasis of early analysis upon an introverted understanding of psychodynamics in terms of a predominantly verbally oriented interpretative method needs to be amplified. While these factors most certainly continue to be of basic significance, they must be balanced by active and guided imaginal work that is nonverbal and bodily. Increasingly we also find that extroverted relationship experiences require attention in order to build an adequate ego structure that eventually may be relativized with respect to the shadow, archetypal world and Self.

Finally, a clear distinction must be made between projection that is a potentially pathological misperception of reality and symbolic experience that mediates our relation to the spirit.

In order to avoid confusing those readers for whom this book serves as an introductory guide, restatements of these subject matters are offered as appendices at the end of the book. ${ }^{1}$

${ }^{1}$ My thanks are due Sylvia Perera for her helpful comments on the proposed changes. 